Journal of Applied Analysis

Vol. 14, No. 2 (2008), pp. 251-257

\title{
ON A REFINEMENT TYPE EQUATION
}

\author{
R. KAPICA and J. MORAWIEC
}

Received June 4, 2007 and, in revised form, July 15, 2008

\begin{abstract}
Let $(\Omega, \mathcal{A}, P)$ be a complete probability space. We show that the trivial function is the unique $L^{1}$-solution of the following refinement type equation

$$
f(x)=\int_{\Omega}\left|\varphi_{x}^{\prime}(x, \omega)\right| f(\varphi(x, \omega)) d P(\omega)
$$

for a wide class of the given functions $\varphi$. This class contains functions of the form $\varphi(x, \omega)=\alpha(\omega) x-\beta(\omega)$ with $-\infty<\int_{\Omega} \log |\alpha(\omega)| d P(\omega)<0$.
\end{abstract}

\section{INTRODUCTION}

Throughout this paper, fix a complete probability space $(\Omega, \mathcal{A}, P)$ and a function $\varphi: \mathbb{R} \times \Omega \rightarrow \mathbb{R}$ satisfying conditions:

$\varphi(\cdot, \omega)$ is a diffeomorphism from $\mathbb{R}$ onto $\mathbb{R}$ for $\omega \in \Omega$,

$\varphi(x, \cdot)$ is a measurable function for $x \in \mathbb{R}$,

$\left(l_{1} \otimes P\right)\left(\varphi^{-1}(B)\right)=0$ for $B \in \mathcal{B}(\mathbb{R})$ with $l_{1}(B)=0$.

2000 Mathematics Subject Classification. Primary: 45A05, 39B12, 42 A38.

Key words and phrases. Refinement type equations, $L^{1}$-solutions, iterates of randomvalued functions, Fourier transform.

This work was supported by Silesian University Mathematics Department (the first author was supported by the Discrete Dynamical Systems and Iteration Theory program, the second author was supported by the Linear Functional Equations program).

ISSN 1425-6908（C) Heldermann Verlag. 
We are interested in $L^{1}$-solutions $f: \mathbb{R} \rightarrow \mathbb{R}$ of the following refinement type equation

$$
f(x)=\int_{\Omega}\left|\varphi_{x}^{\prime}(x, \omega)\right| f(\varphi(x, \omega)) d P(\omega) .
$$

Before we discuss our assumptions notice that if $\alpha: \Omega \rightarrow \mathbb{R} \backslash\{0\}$ and $\beta: \Omega \rightarrow \mathbb{R}$ are measurable functions, then the function $\varphi: \mathbb{R} \times \Omega \rightarrow \mathbb{R}$ given by

$$
\varphi(x, \omega)=\alpha(\omega) x-\beta(\omega)
$$

satisfies conditions (1.1)-(1.3). In this case equation (1.4) takes the form

$$
f(x)=\int_{\Omega}|\alpha(\omega)| f(\alpha(\omega) x-\beta(\omega)) d P(\omega)
$$

and contains the discrete refinement equation $f(x)=\sum_{n \in \mathbb{Z}} c_{n} f(\alpha x-n)$ and the continuous refinement equation $f(x)=\int_{\mathbb{R}} c(y) f(\alpha x-y) d y$, which appear in many areas of pure and applied mathematics (see [4]-[8], [11]-[13], [22], [23]; cf. [9] where more details can be found).

\section{Discussion On ASSUMPTIONS}

Conditions (1.1) and (1.2) imply that both functions $\varphi$ and $\varphi_{x}^{\prime}$ are measurable with respect to the product $\sigma$-algebra $\mathcal{B}(\mathbb{R}) \otimes \mathcal{A}$ (see [15]; cf. [21]). Fix a Lebesgue integrable function $f: \mathbb{R} \rightarrow \mathbb{R}$ and a set $B \in \mathcal{B}(\mathbb{R})$. From (1.3) we see that the set $(f \circ \varphi)^{-1}(B)$ belongs to the completion $\overline{\mathcal{B}(\mathbb{R}) \otimes \mathcal{A}}$ of $\mathcal{B}(\mathbb{R}) \otimes \mathcal{A}$. Consequently, the function $\left|\varphi_{x}^{\prime}(f \circ \varphi)\right|$ is measurable with respect to $\overline{\mathcal{L}_{1} \otimes \mathcal{A}}$, and

$$
\begin{aligned}
\int_{\mathbb{R} \times \Omega}\left|\varphi_{x}^{\prime}(x, \omega) f(\varphi(x, \omega))\right| d\left(\overline{l_{1} \otimes P}\right)(x, \omega) & =\int_{\Omega} \int_{\mathbb{R}}|f(y)| d y d P(\omega) \\
& =\int_{\mathbb{R}}|f(y)| d y<+\infty .
\end{aligned}
$$

(We will need integrability of $\left|\varphi_{x}^{\prime}\right|(f \circ \varphi)$ later.) Since $\mathcal{A}$ is complete we conclude that $\left|\varphi_{x}^{\prime}(x, \cdot)\right|(f \circ \varphi)(x, \cdot)$ is a measurable and integrable function for almost all $x \in \mathbb{R}$ and the integral in (1.4) is a Lebesgue measurable and Lebesgue integrable function of variable $x$.

Fix two Lebesgue integrable functions $f, g: \mathbb{R} \rightarrow \mathbb{R}$ and a set $B \in \mathcal{B}(\mathbb{R})$ of Lebesgue measure zero such that $f(x)=g(x)=$ $\int_{\Omega}\left|\varphi_{x}^{\prime}(x, \omega)\right| g(\varphi(x, \omega)) d P(\omega)$ for $x \notin B$. By (1.3) we have

$$
0=\left(l_{1} \otimes P\right)\left(\varphi^{-1}(B)\right)=\int_{\mathbb{R}} P\left(\varphi^{-1}(B)_{x}\right) d x .
$$


Hence there exists a set $C$ of Lebesgue measure zero such that $P\left(\varphi^{-1}(B)_{x}\right)=0$ for $x \notin C$. Consequently,

$$
\begin{aligned}
f(x) & =\int_{\Omega \backslash \varphi^{-1}(B)_{x}}\left|\varphi_{x}^{\prime}(x, \omega)\right| g(\varphi(x, \omega)) d P(\omega) \\
& =\int_{\Omega}\left|\varphi_{x}^{\prime}(x, \omega)\right| f(\varphi(x, \omega)) d P(\omega)
\end{aligned}
$$

for $x \notin B \cup C$.

Concluding, we have proved the following fact. If $f: \mathbb{R} \rightarrow \mathbb{R}$ is an $L^{1}$ function, then the integral in (1.4) is an $L^{1}$-function of variable $x$ and if a representative of $f$ satisfies (1.4) for almost all $x \in \mathbb{R}$, then $f$ satisfies (1.4) in $L^{1}$-sense. Thus the question on $L^{1}$-solutions of (1.4) is well posed.

It is clear that the set of all $L^{1}$-solutions of (1.4) is a vector subspace of $L^{1}$.

\section{MAIN RESUlts}

Fix $x_{0} \in \mathbb{R}$ and put $\Omega_{1}=\left\{\omega \in \Omega: \varphi_{x}^{\prime}\left(x_{0}, \omega\right)>0\right\}$. From (1.1) it follows that $\Omega_{1}$ does not depend on the choice of $x_{0} \in \mathbb{R}$.

The following proposition is a useful tool for studying the existence of $L^{1}$-solutions of (1.4).

Proposition 3.1. Equation (1.4) has a non-trivial $L^{1}$-solution if and only if the equation

$$
F(t)=\int_{\Omega_{1}} F(\varphi(t, \omega)) d P(\omega)+\int_{\Omega \backslash \Omega_{1}}[1-F(\varphi(t, \omega))] d P(\omega)
$$

has an absolutely continuous probability distribution solution.

Proof. Fix a non-trivial $L^{1}$-solution $f$ of (1.4). Without loss of generality we can assume that $\|f\|_{1}=1$. Since

$$
1=\|f\|_{1} \leq \int_{\mathbb{R}} \int_{\Omega}\left|\varphi_{x}^{\prime}(x, \omega) f(\varphi(x, \omega))\right| d P(\omega) d x=\int_{\mathbb{R}}|f(x)| d x=1,
$$

we have $|f(x)|=\int_{\Omega}\left|\varphi_{x}^{\prime}(x, \omega) f(\varphi(x, \omega))\right| d P(\omega)$, which means that $|f|$ is an $L^{1}$-solution of (1.4). Putting $F(t)=\int_{-\infty}^{t}|f(x)| d x$ we have defined an absolutely continuous probability distribution function and

$$
\begin{aligned}
F(t) & =\int_{-\infty}^{t} \int_{\Omega}\left|\varphi_{x}^{\prime}(x, \omega) f(\varphi(x, \omega))\right| d P(\omega) d x \\
& =\int_{\Omega_{1}} \int_{-\infty}^{\varphi(t, \omega)}|f(y)| d y d P(\omega)+\int_{\Omega \backslash \Omega_{1}} \int_{\varphi(t, \omega)}^{+\infty}|f(y)| d y d P(\omega)
\end{aligned}
$$




$$
=\int_{\Omega_{1}} F(\varphi(t, \omega)) d P(\omega)+\int_{\Omega \backslash \Omega_{1}}[1-F(\varphi(t, \omega))] d P(\omega) .
$$

The converse implication follows from the above calculation.

In the proof of our first result we will iterate functions from $\mathbb{R} \times \Omega$ to $\mathbb{R}$. Having a function $\psi: \mathbb{R} \times \Omega \rightarrow \mathbb{R}$ we define its iterates $\psi^{n}: \mathbb{R} \times \Omega^{\infty} \rightarrow \mathbb{R}$, for $n \in \mathbb{N}$, in the following way

$$
\begin{gathered}
\psi^{1}\left(x, \omega_{1}, \omega_{2}, \ldots\right)=\psi\left(x, \omega_{1}\right), \\
\psi^{n+1}\left(x, \omega_{1}, \omega_{2}, \ldots\right)=\psi\left(\psi^{n}\left(x, \omega_{1}, \omega_{2}, \ldots\right), \omega_{n+1}\right) .
\end{gathered}
$$

This definition of iterates were introduced independently in [3] and [14], and then studied also in [1], [17] and [20]. It turns out that such iterates are useful for instance in solving functional equations (see [2], [16], [18]).

Theorem 3.2. Assume that $|\varphi(x, \omega)-\varphi(y, \omega)| \leq L(\omega)|x-y|$ for $x, y \in \mathbb{R}$, $\omega \in \Omega$ with a measurable function $L: \Omega \rightarrow(0,+\infty)$ such that $-\infty<$ $\int_{\Omega} \log L(\omega) d P(\omega)<0$. If $F: \mathbb{R} \rightarrow \mathbb{R}$ is an uniformly continuous and bounded solution of (3.1) then $F$ is constant.

Proof. Fix a uniformly continuous solution $F: \mathbb{R} \rightarrow[-M, M]$ of (3.1), $x_{0}, y_{0} \in \mathbb{R}, \varepsilon>0$ and choose a $\delta>0$ such that

$$
|F(x)-F(y)| \leq \varepsilon \quad \text { for } x, y \in \mathbb{R} \text { with }|x-y| \leq \delta .
$$

We first observe that by induction we get

$$
\left|\varphi^{n}\left(x_{0}, \omega\right)-\varphi^{n}\left(y_{0}, \omega\right)\right| \leq \prod_{k=1}^{n} L\left(\omega_{k}\right)\left|x_{0}-y_{0}\right|
$$

for $n \in \mathbb{N}, \omega \in \Omega^{\infty}$. The Kolmogorov strong law of large numbers now gives

$$
\lim _{n \rightarrow+\infty}\left(\prod_{k=1}^{n} L\left(\omega_{k}\right)\right)^{\frac{1}{n}}=\exp \left(\int_{\Omega} \log L(\omega) d P(\omega)\right)<1 \quad \text { a.s. }
$$

Hence

$$
\lim _{n \rightarrow+\infty} \prod_{k=1}^{n} L\left(\omega_{k}\right)=0 \quad \text { a.s. }
$$

and so

$$
\lim _{n \rightarrow+\infty}\left|\varphi^{n}\left(x_{0}, \omega\right)-\varphi^{n}\left(y_{0}, \omega\right)\right|=0 \quad \text { a.s. }
$$

By the Egoroff theorem there are $C \in \mathcal{A}^{\infty}$ and $N \in \mathbb{N}$ such that

$$
P^{\infty}\left(\Omega^{\infty} \backslash C\right) \leq \varepsilon \quad \text { and }\left|\varphi^{N}\left(x_{0}, \omega\right)-\varphi^{N}\left(y_{0}, \omega\right)\right| \leq \delta \text { for } \omega \in C .
$$


From (3.1) we conclude that

$$
|F(x)-F(y)| \leq \int_{\Omega}|F(\varphi(x, \omega))-F(\varphi(y, \omega))| d P(\omega) \text { for } x, y \in \mathbb{R} .
$$

Since $\varphi$ is measurable with respect to $\mathcal{B}(\mathbb{R}) \otimes \mathcal{A}$, it follows that its iterates are measurable with respect to $\mathcal{B}(\mathbb{R}) \otimes \mathcal{A}^{\infty}$ (see [3]). Thus we can iterate inequality (3.4). This fact jointly with (3.2) and (3.3) leads to

$$
\begin{aligned}
\left|F\left(x_{0}\right)-F\left(y_{0}\right)\right| \leq & \int_{\Omega^{\infty}}\left|F\left(\varphi^{N}\left(x_{0}, \omega\right)\right)-F\left(\varphi^{N}\left(y_{0}, \omega\right)\right)\right| d P^{\infty}(\omega) \\
\leq & \int_{C}\left|F\left(\varphi^{N}\left(x_{0}, \omega\right)\right)-F\left(\varphi^{N}\left(y_{0}, \omega\right)\right)\right| d P^{\infty}(\omega) \\
& +2 M \int_{\Omega^{\infty} \backslash C} d P^{\infty}(\omega) \leq \varepsilon+2 M \varepsilon
\end{aligned}
$$

which completes the proof.

Note that in the case where $P\left(\Omega_{1}\right)=1$ and $\varphi$ is an affine transformation given by (1.5) with $\alpha>0$ a criterion for nonexistence (existence) of continuous, bounded and nonconstant solutions of (3.1) has been found in [10]. Theorem 3.2 extends the nonexistence part of that result both by considering a more general equation and a wider class of the given function $\varphi$. In fact, it concerns uniformly continuous solutions, but we do not need additional conditions guarantying the convergence of iterates.

From Proposition 3.1 and Theorem 3.2 we get the following result on $L^{1}$-solutions of (1.4).

Theorem 3.3. Under the assumptions of Theorem 3.2 the trivial function is the unique $L^{1}$-solution of (1.4).

If $\varphi$ has form (1.5), then Theorem 3.3 gives the following result on both (discrete and continuous) refinement equations.

Corollary 3.4. Assume that $\alpha: \Omega \rightarrow \mathbb{R} \backslash\{0\}$ and $\beta: \Omega \rightarrow \mathbb{R}$ are measurable functions and

$$
-\infty<\int_{\Omega} \log |\alpha(\omega)| d P(\omega)<0 .
$$

Then the trivial function is the unique $L^{1}$-solution of (1.6). 


\section{References}

[1] Baron, K., On the convergence of sequences on iterates of random-valued functions, Aequationes Math. 32 (1987), 240-251.

[2] Baron, K., Jarczyk, W., Random-valued functions and iterative functional equations, Aequationes Math. 67 (2004), 140-153.

[3] Baron, K., Kuczma, M., Iteration of random-valued functions on the unit interval, Colloq. Math. 37 (1977), 263-269.

[4] Cavaretta, D., Dahmen, W., Micchelli, C. A., Stationary subdivision, Mem. Amer. Math. Soc. 93 (1991), 1-186.

[5] Cohen, A., Daubechies, I., A stability criterion for the orthogonal wavelet bases and their related subband coding scheme, Duke Math. J. 68 (1992), 313-335.

[6] Chui, C. K., Shi, X., Continuous two-scale equations and dyadic wavelets, Adv. Comput. Math. 2 (1994), 185-213.

[7] Dahmen, W., Micchelli, C. A., Continuous refinement equations and subdivision, Adv. Comput. Math. 1 (1993), 1-37.

[8] Daubechies, I., Orthonormal bases of wavelets with compact support, Comm. Pure Appl. Math. 41 (1988), 909-996.

[9] Daubechies, I., Lagarias, J. C., Two-scale difference equations I. Existence and global regularity of solutions, SIAM J. Math. Anal. 22 (1991), 1388-1410.

[10] Derfel, G., Probabilistic method for a class of functional-differential equations (in Russian), Ukrain. Mat. Zh. 41 (1989), 1322-1327, 1436, (translation in Ukrainian Math. J. 41 (1989), 1137-1141 (1990)).

[11] Derfel, G., Dyn, N., Levin, D., Generalized refinement equations and subdivision processes, J. Approx. Theory 80 (1995), 272-297.

[12] Derfel, G., Schilling, R., Spatially chaotic configurations and functional equations with rescaling, J. Phys. A. 29 (1996), 4537-4547.

[13] Deslauriers, G., Dubuc, S., Symmetric iterative interpolation processes. Fractal approximation, Constr. Approx. 5 (1989), 49-68.

[14] Diamond, Ph., A stochastic functional equations, Aequationes Math. 15 (1977), 225233.

[15] Himmelberg, C. J., Measurable relations, Fund. Math. 87 (1975), 53-72.

[16] Kapica, R., Sequences of iterates of random-valued vector functions and continuous solutions of a linear functional equation of infinite order, Bull. Polish Acad. Sci. Math. 50 (2002), 447-455.

[17] Kapica, R., Convergence of sequences of iterates of random-valued vector functions, Colloq. Math. 97 (2003), 1-6.

[18] Kapica, R., Sequences of iterates of random-valued vector functions and solutions of related equations, Österreich. Akad. Wiss. Math.-Natur. Kl. Sitzungsber. II 213 (2004), 113-118 (2005).

[19] Kapica, R., Morawiec, J., Probability distribution functions of the Grincevičjus series, J. Math. Anal. Appl. 342 (2008), 1380-1387.

[20] Kuczma, M., Normalizing factors for iterates of random valued functions, Pr. Nauk. Uniw. Śl. Katow. 6 (1975), 67-72.

[21] Kuratowski, K., Topology. Vol. I (translated from the French by J. Jaworowski), Academic Press, New York-London; PWN, Warsaw, 1966.

[22] Micchelli, C. A., Prautzsch, H., Uniform refinement of curves, Linear Algebra Appl. 114/115 (1989), 841-870.

[23] Schumaker, L. L., Spline functions: Basic theory, John Wiley, New York, 1981. 
RAFA£ KAPICA

Institute of MATHEMATICS

SILESIAN UNIVERSITY

BANKOWA 14

PL-40-007 Katowice, Poland

E-MAIL: RKAPICA@MATH.US.EDU.PL
JANusz MorawiEC Institute OF MATHEMATICS Silesian UNIVERSITY BANKOWA 14 PL-40-007 Katowice, Poland E-MAIL: MORAWIEC@MATH.US.EDU.PL 